\title{
Principally Goldie*-Lifting Modules
}

\author{
Ayşe Tuğba Güroğlu * and Elif Tuğçe Meriç ${ }^{\dagger}$
}

\begin{abstract}
A module $M$ is called principally Goldie*-lifting if for every proper cyclic submodule $X$ of $M$, there is a direct summand $D$ of $M$ such that $X \beta^{*} D$. In this paper, we focus on principally Goldie*-lifting modules as generalizations of lifting modules. Various properties of these modules are given.
\end{abstract}

Mathematics Subject Classification (2010): 16D10, 16D40, 16D70.

Keywords: Principally supplemented, Principally lifting, Goldie*-lifting, Principally Goldie*-lifting .

\section{Introduction}

Throughout this paper $R$ denotes an associative ring with identity and all modules are unital right $R$-modules. $\operatorname{Rad}(M)$ will denote the Jacobson radical of $M$. Let $M$ be an $R$-module and $N, K$ be submodules of $M$. A submodule $K$ of a module $M$ is called small (or superfluous) in $M$, denoted by $K \ll M$, if for every submodule $N$ of $M$ the equality $K+N=M$ implies $N=M . K$ is called a supplement of $N$ in $M$ if $K$ is a minimal with respect to $N+K=M$, equivalently $K$ is a supplement (weak supplement) of $N$ in $M$ if and only if $K+N=M$ and $K \cap N \ll K(K \cap N \ll M)$. A module $M$ is called supplemented module (weakly supplemented module) if every submodule of $M$ has a supplement (weak supplement) in $M$. A module $M$ is $\oplus$-supplemented module if every submodule of $M$ has a supplement which

*Celal Bayar University, Faculty of Arts and Sciences, Department of Mathematics, Muradiye, Manisa, Turkey.

e-mail : tugba.guroglu@cbu.edu.tr

$\dagger$ Celal Bayar University, Faculty of Arts and Sciences, Department of Mathematics, Muradiye, Manisa, Turkey.

e-mail : tugce.meric@cbu.edu.tr 
is a direct summand in $M$. [1] defines principally supplemented modules and investigates their properties. A module $M$ is said to be principally supplemented if for all cyclic submodule $X$ of $M$ there exists a submodule $N$ of $M$ such that $M=N+X$ with $N \cap X$ is small in $N$. A module $M$ is said to be $\oplus$-principally supplemented if for each cyclic submodule $X$ of $M$ there exists a direct summand $D$ of $M$ such that $M=D+X$ and $D \cap X \ll D$. A nonzero module $M$ is said to be hollow if every proper submodule of $M$ is small in $M$. A nonzero module $M$ is said to be principally hollow which means every proper cyclic submodule of $M$ is small in $M$. Clearly, hollow modules are principally hollow. Given submodules $K \subseteq N \subseteq M$ the inclusion $K \stackrel{c s}{\hookrightarrow} N$ is called cosmall in $M$, denoted by $K \hookrightarrow N$, if $N / K \ll M / K$.

Lifting modules play an important role in module theory. Also their various generalizations are studied by many authors in [1], [4], 6], [7, [8], [9], [10] etc. A module $M$ is called lifting module if for every submodule $N$ of $M$ there is a decomposition $M=D \oplus D^{\prime}$ such that $D \subseteq N$ and $D^{\prime} \cap N \ll M$. A module $M$ is called principally lifting module if for all cyclic submodule $X$ of $M$ there exists a decomposition $M=D \oplus D^{\prime}$ such that $D \subseteq X$ and $D^{\prime} \cap X \ll M$. G.F.Birkenmeier et.al. [4] defines $\beta^{*}$ relation to study on the open problem 'Is every $H$-supplemented module supplemented?' in [8]. They say submodules $X, Y$ of $M$ are $\beta^{*}$ equivalent, $X \beta^{*} Y$, if and only if $\frac{X+Y}{X}$ is small in $\frac{M}{X}$ and $\frac{X+Y}{Y}$ is small in $\frac{M}{Y} . M$ is called Goldie ${ }^{*}$-lifting (or briefly, $\mathcal{G}^{*}$-lifting) if and only if for each $X \leq M$ there exists a direct summand $D$ of $M$ such that $X \beta^{*} D . \quad M$ is called Goldie*-supplemented (or briefly, $\mathcal{G}^{*}$-supplemented) if and only if for each $X \leq M$ there exists a supplement submodule $S$ of $M$ such that $X \beta^{*} S$ (see [4]).

In Section 2, we recall the equivalence relation $\beta^{*}$ which is defined in [4] and investigate some basic properties of it.

In section 3 we define principally Goldie*-lifting modules as a generalization of lifting modules. We give some neccesary assumptions for a quotient module or a direct summand of a principally Goldie*-lifting module to be principally Goldie*-lifting. Principally lifting, principally Goldie*-lifting and principally supplemented modules are compared. It is also shown that principally lifting, principally Goldie*-lifting and $\oplus$-principally supplemented coincide on $\pi$-projective modules. 


\section{Properties of $\beta^{*}$ Relation}

Definition 2.1. (See [4) Any submodules $X, Y$ of $M$ are $\beta^{*}$ equivalent, $X \beta^{*} Y$, if and only if $\frac{X+Y}{X}$ is small in $\frac{M}{X}$ and $\frac{X+Y}{Y}$ is small in $\frac{M}{Y}$.

Lemma 2.2. (See 4]) $\beta^{*}$ is an equivalence relation.

By [4], page 43], the zero submodule is $\beta^{*}$ equivalent to any small submodule.

Theorem 2.3. (See [4]) Let $X, Y$ be submodules of $M$. The following are equivalent:

(a) $X \beta^{*} Y$.

(b) $X \stackrel{c s}{\hookrightarrow} X+Y$ and $Y \stackrel{c s}{\hookrightarrow} X+Y$.

(c) For each submodule $A$ of $M$ such that $X+Y+A=M$, then $X+A=M$ and $Y+A=M$.

(d) If $K+X=M$ for any submodule $K$ of $M$, then $Y+K=M$ and if $Y+H=M$ for any submodule $H$ of $M$, then $X+H=M$.

Lemma 2.4. Let $M=D \oplus D^{\prime}$ and $A, B \leq D$. Then $A \beta^{*} B$ in $M$ if and only if $A \beta^{*} B$ in $D$.

Proof. $(\Rightarrow)$ Let $A \beta^{*} B$ in $M$ and $A+B+N=D$ for some submodule $N$ of $D$. Let us show $A+N=D$ and $B+N=D$. Since $A \beta^{*} B$ in $M$,

$$
M=D \oplus D^{\prime}=A+B+N+D^{\prime}
$$

implies $A+N+D^{\prime}=M$ and $B+N+D^{\prime}=M$. By [[11], 41], $A+N=D$ and $B+N=D$. From Theorem 2.3, we get $A \beta^{*} B$ in $D$.

$(\Leftarrow)$ Let $A \beta^{*} B$ in $D$. Then $\frac{A+B}{A} \ll \frac{D}{A}$ implies $\frac{A+B}{A} \ll \frac{M}{A}$. Similarly, $\frac{A+B}{B} \ll \frac{D}{B}$ implies $\frac{A+B}{B} \ll \frac{M}{B}$. This means that $A \beta^{*} B$ in $M$.

Lemma 2.5. If a direct summand $D$ in $M$ is $\beta^{*}$ equivalent to a cyclic submodule $X$ of $M$, then $D$ is also cyclic.

Proof. Assume that $M=D \oplus D^{\prime}$ for some submodules $D, D^{\prime}$ of $M$ and $X$ is a cyclic submodule of $M$ which is $\beta^{*}$ equivalent to $D$. By Theorem 2.3, $M=X+D^{\prime}$. Since $\frac{X+D^{\prime}}{D^{\prime}}=\frac{M}{D^{\prime}} \cong D, D$ is cyclic. 


\section{Principally Goldie* - Lifting Modules}

In [4], the authors defined $\beta^{*}$ relation and they introduced two notions called Goldie*-supplemented module and Goldie*-lifting module depend on $\beta^{*}$ relation. $M$ is called Goldie ${ }^{*}$-lifting (or briefly, $\mathcal{G}^{*}$-lifting) if and only if for each $N \leq M$ there exists a direct summand $D$ of $M$ such that $N \beta^{*} D$. $M$ is called Goldie*-supplemented (or briefly, $\mathcal{G}^{*}$-supplemented) if and only if for each $N \leq M$ there exists a supplement submodule $S$ of $M$ such that $N \beta^{*} S$. A module $M$ is said to be $H$-supplemented if for every submodule $N$ there is a direct summand $D$ of $M$ such that $M=N+B$ holds if and only if $M=D+B$ for any submodule $B$ of $M$. They showed that Goldie*-lifting modules and $H$-supplemented modules are the same in [[4], Theorem 3.6]. In this section, we define principally Goldie*-lifting module (briefly principally $\mathcal{G}^{*}$-lifting module) as a generalization of $\mathcal{G}^{*}$-lifting module and investigate some properties of this module.

Definition 3.1. A module $M$ is called principally Goldie*-lifting module (briefly principally $\mathcal{G}^{*}$-lifting) if for each cyclic submodule $X$ of $M$, there exists a direct summand $D$ of $M$ such that $X \beta^{*} D$.

Clearly, every $\mathcal{G}^{*}$-lifting module is principally $\mathcal{G}^{*}$-lifting. However the converse does not hold.

Example 3.2. Consider the $\mathbb{Z}$-module $\mathbb{Q}$. Since $\operatorname{Rad}(\mathbb{Q})=\mathbb{Q}$, every cyclic submodule of $\mathbb{Q}$ is small in $\mathbb{Q}$. By [4], Example 2.15], the $\mathbb{Z}$-module $\mathbb{Q}$ is principally $\mathcal{G}^{*}$-lifting. But the $\mathbb{Z}$-module $\mathbb{Q}$ is not supplemented. So it is not $\mathcal{G}^{*}$-lifting by [[4], Theorem 3.6].

A module $M$ is said to be radical if $\operatorname{Rad}(M)=M$.

Lemma 3.3. Every radical module is principally $\mathcal{G}^{*}$-lifting.

Proof. Let $m \in M$. If $M$ is radical, $m R \subseteq \operatorname{Rad}(M)$. By [[11], 21.5], $m R \ll$ $M$. So we get $m R \beta^{*} 0$. Thus $M$ is principally $\mathcal{G}^{*}$-lifting.

Theorem 3.4. Let $M$ be a module. Consider the following conditions:

(a) $M$ is principally lifting,

(b) $M$ is principally $\mathcal{G}^{*}$-lifting,

(c) $M$ is principally supplemented.

Then $(a) \Rightarrow(b) \Rightarrow(c)$. 
Proof. $(a) \Rightarrow(b)$ Let $m \in M$. Then $m R$ is cyclic submodule of $M$. From (a), there is a decomposition $M=D \oplus D^{\prime}$ with $D \leq m R$ and $m R \cap D^{\prime} \ll M$. Since $D \leq m R, \frac{m R+D}{m R} \ll \frac{M}{m R}$. By modularity, $m R=D \oplus\left(m R \cap D^{\prime}\right)$. Then $\frac{m R}{D} \cong m R \cap D^{\prime}$ and $\frac{M}{D} \cong D^{\prime}$. If $m R \cap D^{\prime} \ll M$, by [[11], 19.3], $m R \cap D^{\prime} \ll D^{\prime}$. It implies that $\frac{m R+D}{D} \ll \frac{M}{D}$. Therefore it is seen that $m R \beta^{*} D$ from Definition 2.1. Hence $M$ is principally $\mathcal{G}^{*}$-lifting.

$(b) \Rightarrow(c)$ Let $m \in M$. By hypothesis, there exists a direct summand $D$ of $M$ such that $m R \beta^{*} D$. Since $M=D \oplus D^{\prime}$ for some submodule $D^{\prime}$ of $M$ and $D^{\prime}$ is a supplement of $D, D^{\prime}$ is a supplement of $m R$ in $M$ by [[4], Theorem 2.6(ii)]. Thus $M$ is principally supplemented.

The following example shows that a principally $\mathcal{G}^{*}$-lifting module need not be principally lifting in general:

Example 3.5. Consider the $\mathbb{Z}$-module $M=\mathbb{Z} / 2 \mathbb{Z} \oplus \mathbb{Z} / 8 \mathbb{Z}$. By [[10], Example 3.7], $M$ is $H$-supplemented module. Then $M$ is $\mathcal{G}^{*}$-lifting by [4], Theorem 3.6]. Since every $\mathcal{G}^{*}$-lifting module is principally $\mathcal{G}^{*}$-lifting, $M$ is principally $\mathcal{G}^{*}$-lifting. But from [[1], Examples $\left.7 .(3)\right], M$ is not principally lifting.

Theorem 3.6. Let $M$ be an indecomposable module. Consider the following conditions:

(a) $M$ is principally lifting,

(b) $M$ is principally hollow,

(c) $M$ is principally $\mathcal{G}^{*}$-lifting.

Then $(a) \Leftrightarrow(b) \Leftrightarrow(c)$

Proof. $(a) \Leftrightarrow(b)$ It is easy to see from [1], Lemma 14].

$(b) \Rightarrow(c)$ Let $M$ be principally hollow and $m \in M$. Then $m R \ll M$ implies that $m R \beta^{*} 0$.

$(c) \Rightarrow(b)$ Let $m R$ be a proper cyclic submodule of $M$. By $(c)$, there exists a decomposition $M=D \oplus D^{\prime}$ such that $m R \beta^{*} D$. Since $M$ is indecomposable, $D=M$ or $D=0$. Let $D=M$. From [4], Corollary 2.8.(iii)], we obtain $m R=M$ but this is a contradiction. Thus $m R \beta^{*} 0$ and so $m R \ll M$. That is, $M$ is principally hollow. 
We shall give the following example of modules which are principally supplemented but not principally $\mathcal{G}^{*}$-lifting.

Example 3.7. Let $F$ be a field, $x$ and $y$ commuting indeterminates over $F$. Let $R=F[x, y]$ be a polynomial ring and its ideals $I_{1}=\left(x^{2}\right)$ and $I_{2}=\left(y^{2}\right)$ and the ring $S=R /\left(x^{2}, y^{2}\right)$. Consider the $S$-module $M=\bar{x} S+\bar{y} S$. By [[1], Example 15], $M$ is an indecomposable $S$-module and it is not principally hollow. Then $M$ is not principally $\mathcal{G}^{*}$-lifting from Theorem 3.6. Again by [[1], Example 15], $M$ is principally supplemented.

A module $M$ is said to be principally semisimple if every cyclic submodule of $M$ is a direct summand of $M$.

Lemma 3.8. Every principally semisimple module is principally $\mathcal{G}^{*}$-lifting.

Proof. It is clear from the definition of semisimple modules and the reflexive property of $\beta^{*}$.

Recall that a submodule $N$ is called fully invariant if for each endomorphism $f$ of $M, f(N) \leq N$. A module $M$ is said to be duo module if every submodule of $M$ is fully invariant. A module $M$ is called distributive if for all submodules $A, B, C$ of $M, A+(B \cap C)=(A+B) \cap(A+C)$ or $A \cap(B+C)=(A \cap B)+(A \cap C)$.

Proposition 3.9. Let $M=M_{1} \oplus M_{2}$ be a duo module (or distributive module). Then $M$ is principally $\mathcal{G}^{*}$-lifting if and only if $M_{1}$ and $M_{2}$ are principally $\mathcal{G}^{*}$-lifting.

Proof. $(\Rightarrow)$ Let $m \in M_{1}$. Since $M$ is principally $\mathcal{G}^{*}$-lifting, there is a decomposition $M=D \oplus D^{\prime}$ such that $m R \beta^{*} D$ in $M$. As $M$ is duo module $M_{1}=\left(M_{1} \cap D\right) \oplus\left(M_{1} \cap D^{\prime}\right), m R=(m R \cap D) \oplus\left(m R \cap D^{\prime}\right)$ and $D^{\prime}=\left(M_{1} \cap D^{\prime}\right) \cap\left(M_{2} \cap D^{\prime}\right)$. We claim that $m R \beta^{*}\left(M_{1} \cap D\right)$ in $M_{1}$. Since $\frac{m R+\left(M_{1} \cap D\right)}{m R} \leq \frac{m R+D}{m R}$ and $\frac{m R+D}{m R} \ll \frac{M}{m R}$, we have $\frac{m R+\left(M_{1} \cap D\right)}{m R} \ll \frac{M}{m R}$ by [[11], 19.3(2)]. From isomorphism theorem and the direct decomposition of $m R$

$$
\frac{m R+\left(M_{1} \cap D\right)}{M_{1} \cap D} \cong \frac{m R}{m R \cap\left(M_{1} \cap D\right)}=\frac{m R}{m R \cap D} \cong m R \cap D^{\prime} .
$$

Since $D^{\prime}$ is a supplement of $m R, m R \cap D^{\prime} \ll D^{\prime}$. By [[11], 19.3(5)], $m R \cap D^{\prime} \ll M_{1} \cap D^{\prime}$. Further $M_{1} \cap D^{\prime} \cong \frac{M_{1}}{M_{1} \cap D}$. This shows that 
$\frac{m R+\left(M_{1} \cap D\right)}{M_{1} \cap D}$ is small in $\frac{M_{1}}{M_{1} \cap D}$ and also in $\frac{M}{M_{1} \cap D}$. From Definition 2.1 we get $m R \beta^{*}\left(M_{1} \cap D\right)$ in $M$. Then $m R \beta^{*}\left(M_{1} \cap D\right)$ in $M_{1}$ by Lemma 2.4.

$(\Leftarrow)$ Let $m \in M$. If $M$ is a duo module, for cyclic submodule $m R$ of $M$, $m R=\left(m R \cap M_{1}\right) \oplus\left(m R \cap M_{2}\right)$. So $m R \cap M_{1}=m_{1} R$ and $m R \cap M_{2}=m_{2} R$ for some $m_{1} \in M_{1}, m_{2} \in M_{2}$. Since $M_{1}$ and $M_{2}$ are principally $\mathcal{G}^{*}$-lifting, there are decompositions $M_{1}=D_{1} \oplus D_{1}^{\prime}$ and $M_{2}=D_{2} \oplus D_{2}^{\prime}$ such that $m_{1} R \beta^{*} D_{1}$ in $M_{1}$ and $m_{2} R \beta^{*} D_{2}$ in $M_{2}$. By Lemma 2.4, $m_{1} R \beta^{*} D_{1}$ and $m_{2} R \beta^{*} D_{2}$ in $M$. Since $m R=m_{1} R+m_{2} R$, by [4], Proposition 2.11], $m R \beta^{*}\left(D_{1} \oplus D_{2}\right)$.

Proposition 3.10. Let any cyclic submodule of $M$ have a supplement which is a relatively projective direct summand of $M$. Then $M$ is principally $\mathcal{G}^{*}$-lifting.

Proof. Let $m \in M$. By hypothesis, there exsists a decomposition $M=D \oplus D^{\prime}$ such that $M=m R+D^{\prime}$ and $m R \cap D^{\prime} \ll D^{\prime}$. Because $D$ is $D^{\prime}$-projective, $M=A \oplus D^{\prime}$ for some submodule $A$ of $m R$ by [[]], Lemma 4.47]. So $M$ is principally lifting. It follows from Theorem 3.4 that $\mathrm{M}$ is principally $\mathcal{G}^{*}$-lifting.

Proposition 3.11. Let $M$ be principally $\mathcal{G}^{*}$-lifting and $N$ be a submodule of $M$. If $\frac{N+D}{N}$ is a direct summand in $\frac{M}{N}$ for any cyclic direct summand $D$ of $M$, then $\frac{M}{N}$ is principally $\mathcal{G}^{*}$-lifting.

Proof. Let $\frac{m R+N}{N}$ be a cyclic submodule of $\frac{M}{N}$ for $m \in M$. Since $M$ is principally $\mathcal{G}^{*}$-lifting there exists a decomposition $M=D \oplus D^{\prime}$ such that $m R \beta^{*} D$. Then $D$ is also cyclic Lemma 2.5. By hypothesis, $\frac{D+N}{N}$ is a direct summand in $\frac{M}{N}$. We claim that $\frac{m R+N}{N} \beta^{*} \frac{D+N}{N}$. Consider the canonical epimorphism $\theta: M \rightarrow M / N$. By [[4], Proposition 2.9(i)], $\theta(m R) \beta^{*} \theta(D)$, that is, $\frac{m R+N}{N} \beta^{*} \frac{D+N}{N}$. Thus $\frac{M}{N}$ is principally $\mathcal{G}^{*}$-lifting.

Corollary 3.12. Let $M$ be principally $\mathcal{G}^{*}$-lifting.

(a) If $M$ is distributive (or duo) module, then any quotient module of $M$ principally $\mathcal{G}^{*}$-lifting.

(b) Let $N$ be a projection invariant, that is, $e N \subseteq N$ for all $e^{2}=e \in$ $\operatorname{End}(M)$. Then $\frac{M}{N}$ is principally $\mathcal{G}^{*}$-lifting. In particular, $\frac{M}{A}$ is principally $\mathcal{G}^{*}$-lifting for every fully invariant submodule $A$ of $M$. 
Proof. (a) Let $N$ be any submodule of $M$ and $M=D \oplus D^{\prime}$ for some submodules $D, D^{\prime}$ of $M$. Then

$$
\frac{M}{N}=\frac{D \oplus D^{\prime}}{N}=\frac{D+N}{N}+\frac{D^{\prime}+N}{N} .
$$

Since $M$ is distributive, $N=(D+N) \cap\left(D^{\prime}+N\right)$. We obtain $\frac{M}{N}=\frac{D+N}{N} \oplus \frac{D^{\prime}+N}{N}$. By Theorem 3.11, $\frac{M}{N}$ is principally $\mathcal{G}^{*}$-lifting.

(b) Assume that $M=D \oplus D^{\prime}$ for some $D, D^{\prime} \leq M$. For the projection $\operatorname{map} \pi_{D}: M \rightarrow D, \pi_{D}^{2}=\pi \in \operatorname{End}(M)$ and $\pi_{D}(N) \subseteq N$. So $\pi_{D}(N)=N \cap D$. Similarly, $\pi_{D^{\prime}}(N)=N \cap D^{\prime}$ for the projection map $\pi_{D^{\prime}}: M \rightarrow D^{\prime}$. Hence we have $N=(N \cap D)+\left(N \cap D^{\prime}\right)$. By modularity,

$$
M=\left[D+(N \cap D)+\left(N \cap D^{\prime}\right)\right]+\left(D^{\prime}+N\right)=\left[D \oplus\left(N \cap D^{\prime}\right)\right]+\left(D^{\prime}+N\right) .
$$

and

$\left[D \oplus\left(N \cap D^{\prime}\right)\right] \cap\left(D^{\prime}+N\right)=\left[D \cap\left(D^{\prime}+N\right)\right]+\left(N \cap D^{\prime}\right)=(N \cap D)+\left(N \cap D^{\prime}\right)=N$

Thus $\frac{M}{N}=\frac{D \oplus\left(N \cap D^{\prime}\right)}{N} \oplus \frac{D^{\prime}+N}{N}$. By Theorem 3.11, $\frac{M}{N}$ is principally $\mathcal{G}^{*}$-lifting.

Another consequence of Proposition 3.10 is given in the next result.

A module $M$ is said to have the summand sum property (SSP) if the sum of any two direct summands of $M$ is again a direct summand.

Proposition 3.13. Let $M$ be a principally $\mathcal{G}^{*}$-lifting module. If $M$ has SSP, then any direct summand of $M$ is principally $\mathcal{G}^{*}$-lifting.

Proof. Let $M=N \oplus N^{\prime}$ for some submodule $N, N^{\prime}$ of $M$. Take any cyclic direct summand $D$ of $M$. Since $M$ has SSP, $M=\left(D+N^{\prime}\right) \oplus T$ for some submodule $T$ of $M$. Then

$$
\frac{M}{N^{\prime}}=\frac{D+N^{\prime}}{N^{\prime}}+\frac{T+N^{\prime}}{N^{\prime}}
$$

By modularity,

$$
\left(D+N^{\prime}\right) \cap\left(T+N^{\prime}\right)=N^{\prime}+\left[\left(D+N^{\prime}\right) \cap T\right]=N^{\prime} .
$$

So we obtain

$$
\frac{M}{N^{\prime}}=\frac{D+N^{\prime}}{N^{\prime}} \oplus \frac{T+N^{\prime}}{N^{\prime}} .
$$

Thus $N$ is principally $\mathcal{G}^{*}$-lifting from Proposition 3.11 
Next we show when $M / \operatorname{Rad}(M)$ is principally semisimple in case $M$ is principally $\mathcal{G}^{*}$-lifting module.

Proposition 3.14. Let $M$ be principally $\mathcal{G}^{*}$-lifting and distributive module. Then $\frac{M}{\operatorname{Rad}(M)}$ is a principally semisimple.

Proof. Let $m \in M$. There exists a decomposition $M=D \oplus D^{\prime}$ such that $m R \beta^{*} D$. By [4], Theorem 2.6(ii)], $D^{\prime}$ is a supplement of $m R$, that is, $M=m R+D^{\prime}$ and $m R \cap D^{\prime} \ll D^{\prime}$. Then

$$
\frac{M}{\operatorname{Rad}(M)}=\frac{m R+\operatorname{Rad}(M)}{\operatorname{Rad}(M)}+\frac{D^{\prime}+\operatorname{Rad}(M)}{\operatorname{Rad}(M)} .
$$

Because $M$ is distributive and $m R \cap D^{\prime} \ll D^{\prime}$,

$$
(m R+\operatorname{Rad}(M)) \cap\left(D^{\prime}+\operatorname{Rad}(M)\right)=\left(m R \cap D^{\prime}\right)+\operatorname{Rad}(M)=\operatorname{Rad}(M) .
$$

Hence $\frac{m R+\operatorname{Rad}(M)}{\operatorname{Rad}(M)}$ is a direct summand in $\frac{M}{\operatorname{Rad}(M)}$, this means that $\frac{M}{\operatorname{Rad}(M)}$ is a principally semisimple module.

Proposition 3.15. Let $M$ be a principally $\mathcal{G}^{*}$-lifting module and $\operatorname{Rad}(M) \ll M$. Then $\frac{M}{\operatorname{Rad}(M)}$ is principally semisimple.

Proof. Let $\frac{X}{\operatorname{Rad}(M)}$ be a cyclic submodule of $\frac{M}{\operatorname{Rad}(M)}$. Then $X=m R+$ $\operatorname{Rad}(M)$ for some $m \in M$. There exists a decomposition $M=D \oplus D^{\prime}$ such that $m R \beta^{*} D$. By [4], Theorem 2.6(ii)], $D^{\prime}$ is a supplement of $m R$ in $M$. It follows from [4], Corollary 2.12] that $(m R+\operatorname{Rad}(M)) \beta^{*} D$. Therefore

$$
\frac{M}{\operatorname{Rad}(M)}=\frac{X}{\operatorname{Rad}(M)}+\frac{D^{\prime}+\operatorname{Rad}(M)}{\operatorname{Rad}(M)} .
$$

By modularity and $X \cap D^{\prime} \subseteq \operatorname{Rad}(M)$,

$$
\frac{X}{\operatorname{Rad}(M)} \cap \frac{D^{\prime}+\operatorname{Rad}(M)}{\operatorname{Rad}(M)}=\frac{\left(X \cap D^{\prime}\right)+\operatorname{Rad}(M)}{\operatorname{Rad}(M)} .
$$

Then we obtain that $\frac{M}{\operatorname{Rad}(M)}=\frac{X}{\operatorname{Rad}(M)} \oplus \frac{D^{\prime}+\operatorname{Rad}(M)}{\operatorname{Rad}(M)}$. 
Proposition 3.16. Let $M$ be principally $\mathcal{G}^{*}$-lifting. If $\operatorname{Rad}(M) \ll M$, then $M=A \oplus B$ where $A$ is principally semisimple and $\operatorname{Rad}(B) \ll B$.

Proof. Let $A$ be a submodule of $M$ such that $\operatorname{Rad}(M) \oplus A$ is small in $M$ and $m \in A$. By hypothesis, there exists a decomposition $M=D \oplus D^{\prime}$ such that $m R \beta^{*} D$. Then $D$ is cyclic from Lemma 2.5. By [4], Theorem 2.6(ii)], $M=m R+D^{\prime}$ and $m R \cap D^{\prime} \ll D^{\prime}$. So $m R \cap D^{\prime}=0$ because $m R \cap D^{\prime}$ is a submodule of $\operatorname{Rad}(M)$. That is, $M=m R \oplus D^{\prime}$. Hence $m R=D$. Since $D \cap \operatorname{Rad}(M)=0, D$ is isomorphic to a submodule of $\frac{M}{\operatorname{Rad}(M)}$. By Proposition 3.15, $\frac{M}{\operatorname{Rad}(M)}$ is principally semisimple. Thus $D$ is principally semisimple.

In general, it is not true that principally lifting and principally $\mathcal{G}^{*}$-lifting modules coincide. As we will see the following theorem, we need projectivity condition.

Proposition 3.17. Let $M$ be a $\pi$-projective module. The following are equivalent:

(a) $M$ is principally lifting,

(b) $M$ is principally $\mathcal{G}^{*}$-lifting,

(c) $M$ is $\oplus$-principally supplemented.

Proof. $(a) \Rightarrow(b)$ follows from Theorem 3.4

$(b) \Rightarrow(c)$ follows from [[4, Theorem 2.6(ii)].

$(c) \Rightarrow(a)$ Consider any $m \in M$. From $(c), m R$ has a supplement $D$ which is a direct summand in $M$, that is, $M=m R+D$ and $m R \cap D \ll D$. Since $M$ is $\pi$-projective there exists a complement $D^{\prime}$ of $D$ such that $D^{\prime} \subseteq m R$ by [[5], 4.14(1)]. Thus $M$ is principally lifting.

Proposition 3.18. Let $M$ be a $\pi$-projective module. Then $M$ is principally $\mathcal{G}^{*}$-lifting if and only if every cyclic submodule $X$ of $M$ can be written as $X=D \oplus A$ such that $D$ is a direct summand in $M$ and $A \ll M$.

Proof. $(\Rightarrow)$ Let $M$ be principally $\mathcal{G}^{*}$-lifting and $\pi$-projective module. By Proposition 3.17, $M$ is principally lifting. Then for any cyclic submodule $X$ of $M$ there exists a direct decomposition $M=D \oplus D^{\prime}$ such that $D \leq X$ and $X \cap D^{\prime} \ll M$. By modularity, we conclude that $X=D \oplus\left(X \cap D^{\prime}\right)$.

$(\Leftarrow)$ By assumption and [[6], Lemma 2.10], $M$ is principally lifting. Hence from Proposition $3.17 M$ is principally $\mathcal{G}^{*}$-lifting. 
Now we mention that principally $\mathcal{G}^{*}$-lifting and $\mathcal{G}^{*}$-lifting modules are coincide under some condition.

Proposition 3.19. Let $M$ be Noetherian and have SSP. Then $M$ is principally $\mathcal{G}^{*}$-lifting if and only if $M$ is $\mathcal{G}^{*}$-lifting.

Proof. $(\Leftarrow)$ Clear.

$(\Rightarrow)$ If $M$ is Noetherian, for any submodule $X$ of $M$ there exist some $m_{1}, m_{2}, \ldots, m_{n} \in M$ such that $X=m_{1} R+m_{2} R+\ldots+m_{n} R$ by [[11], 27.1]. Since $M$ is principally $\mathcal{G}^{*}$-lifting, there exist some direct summands $D_{1}, D_{2}, \ldots, D_{n}$ of $M$ such that $m_{1} R \beta^{*} D_{1}, m_{2} R \beta^{*} D_{2}, \ldots, m_{n} R \beta^{*} D_{n} . D=D_{1}+D_{2}+\ldots+D_{n}$ is also a direct summand in $M$ because of SSP. By [[4], Proposition 2.11], $X \beta^{*} D$. Hence $M$ is $\mathcal{G}^{*}$-lifting.

Proposition 3.20. Let any submodule $N$ of $M$ be the sum of a cyclic submodule $X$ and a small submodule $A$ in $M$. Then $M$ is principally $\mathcal{G}^{*}$-lifting if and only if $M$ is $\mathcal{G}^{*}$-lifting.

Proof. $(\Leftarrow)$ Clear.

$(\Rightarrow)$ Let $N=X+A$ for a cyclic submodule $X$ and a small submodule $A$ of $M$. Since $M$ is principally $\mathcal{G}^{*}$-lifting, there exists a direct summand $D$ of $M$ such that $X \beta^{*} D$. From [4], Corollary 2.12], $(X+A) \beta^{*} D$, that is, $N \beta^{*} D$. Hence $M$ is $\mathcal{G}^{*}$-lifting. 


\section{References}

[1] Acar, U., Harmanci, A., Principally Supplemented Modules, Albanian Journal of Math., Vol 4, 3, (2010), 79-88.

[2] Al-Khazzi, I., Smith, P. F., Modules with Chain Condition on Superfluous Submodules, Comm. Algebra, 19(8), (1991), 2331-2351.

[3] Anderson, F.W., Fuller, K.R. Rings and Categories of Modules, Springer, New York, (1992).

[4] Birkenmeier, G.F., Mutlu, F.T., Nebiyev, C., Sokmez, N., Tercan, A., Goldie*-supplemented Modules, Glasgow Math. Journal, 52(A), (2010), 41-52.

[5] Clark, J., Lomp, C., Vanaja, N., Wisbauer, R., Lifting Modules: Supplements and Projectivity in Module Theory, Birkhäuser-Verlag, Basel, Switzerland (2006).

[6] Kamal, M., Yousef A., Principally Lifting Modules, Int. Electron. J.Algebra, 2, (2007), 127-137.

[7] Kosan, T., Tutuncu Keskin D., H-supplemented Duo Modules, Journal of Algebra and Its Applications, Vol. 6, No. 6, (2007), 965-971.

[8] Mohamed. S., Muller, B.J., Continuous and Discrete Modules, Cambridge University Press, (1990).

[9] Talebi, Y., Hamzekolaee, A.R., Tercan, A., Goldie-Rad-supplemented Modules, An. St. Univ. Ovidius Constanta, (2014).

[10] Yongduo, W., Dejun, W., On H-supplemented Modules, Comm. in Algebra, 40, (2012), 3679-3689.

[11] Wisbauer, R., Foundations of Module and Ring Theory, Gordon and Breach, (1991). 